\title{
Association of Impaired Fasting Blood Glucose With Triple Coronary Artery Stenosis and Myocardial Infarction Among Patients With Coronary Artery Stenosis
}

OPEN ACCESS

Edited by:

Wanling Xuan,

University of South Florida,

United States

Reviewed by:

Xina Xie,

Shenzhen Second People's Hospital,

China

Yuli Huang,

Southern Medical University, China

Xiangyuan Pu,

Zhejiang University, China

*Correspondence:

Xuerui Tan

tanxueru i@vip.sina.com

Shu Ye

s_ye@stu.edu.cn

tThese authors have contributed equally to this work and share first

authorship

Specialty section:

This article was submitted to

Stem Cell Research,

a section of the journal

Frontiers in Cell and Developmental

Biology

Received: 22 November 2021

Accepted: 10 February 2022

Published: 03 March 2022

Citation:

Chen C, Chen Y, Xiao J, Zhang Y, Yang Z, Yang $P$, Lu N, Yi K, Chen $X$, Chen S, O'Gara MSC MC, O'Meara M, Ye S and Tan X (2022) Association of Impaired Fasting Blood Glucose With

Triple Coronary Artery Stenosis and Myocardial Infarction Among Patients

With Coronary Artery Stenosis.

Front. Cell Dev. Biol. 10:820124.

doi: 10.3389/fcell.2022.820124
Chang Chen ${ }^{1+}$, Yequn Chen ${ }^{1 \dagger}$, Jiaxin Xiao ${ }^{1+}$, Yanhong Zhang ${ }^{1}$, Zhaorui Yang ${ }^{1}$, Peixuan Yang ${ }^{1}$, Nan Lu ${ }^{1}$, Kaihong Yi ${ }^{1}$, Xiaojun Chen ${ }^{1}$, Shaoxin Chen ${ }^{1}$, Mary Clare O'Gara MSc ${ }^{1}$, Michael O'Meara ${ }^{1}$, Shu Ye ${ }^{1,2,3 *}$ and Xuerui Tan $^{1,2 *}$

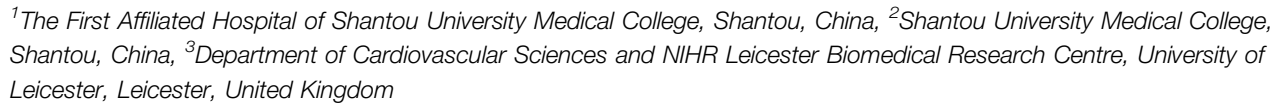

${ }^{1}$ The First Affiliated Hospital of Shantou University Medical College, Shantou, China, ${ }^{2}$ Shantou University Medical College, Shantou, China, ${ }^{3}$ Department of Cardiovascular Sciences and NIHR Leicester Biomedical Research Centre, University of Leicester, Leicester, United Kingdom

Background: The association between impaired fasting glucose level (IFG) and coronary heart disease (CAD) remain controversial. In the present study, we sought to ascertain a relationship of IFG with the number of diseased coronary artery and occurrence of myocardial infarction, among CAD cases.

Methods: We studied 1,451 consecutive no-diabetic patients who underwent coronary angiography at the First Affiliated Hospital of Shantou University Medical College in Southern China. Demographic, biochemical, clinical and angiographic data were collected.

Results: The prevalence of IFG was higher in patients with angiographically confirmed CAD than in subjects without angiographic evidence of CAD (33.4 versus 28.2\%, $p=0.034$ ). Compared with CAD cases without IFG, CAD cases with IFG had a higher odds ratio (OR) of having triple-vessel disease as opposed to having single- or double-vessel disease $[\mathrm{OR}=1.53$, 95\% confidence interval $(\mathrm{Cl})=1.13-2.07]$. Furthermore, the occurrence of Ml was higher in CAD cases with IFG than in CAD cases without IFG (OR $=1.73,95 \% \mathrm{Cl}=1.27-2.36)$.

Conclusions: There is an association between IFG and a predisposition to severe CAD indicated by triple vessel disease or myocardial infarction.

Keywords: impaired fasting blood glucose, coronary artery disease, myocardial infarction, triple-vessel disease, single- or double-vessel disease

\section{INTRODUCTION}

Many epidemiological studies have shown that individuals of diabetes mellitus are more likely to have coronary artery disease (CAD) than no-diabetic individuals (Kannel and McGee, 1979; Martins et al., 2015). It is now widely accepted that diabetes mellitus is a major risk factor for CAD.

Impaired fasting glucose (also known as impaired fasting glycemia, IFG) is a type of prediabetes. In individuals with IFG, fasting blood sugar levels are consistently above the normal range but below 
the threshold for a diagnosis of diabetes mellitus. IFG has been associated with micro vascular disease (Hanna-Moussa et al., 2009), heart failure and cardiovascular mortality (Henry et al., 2002; Sorkin et al., 2005; Xu et al., 2015; Huang et al., 2016; Cai et al., 2020; Cai et al., 2021; Mai et al., 2021) Several meta-analyses concluded that IFG was associated with an increased risk of CAD. However, it is still unclear as to whether or not there is a relationship between IFG and severity of CAD (Yan et al., 2009; Konstantinou et al., 2010), and further investigations are required.

In the present study, we examined a cohort of non-diabetic individuals undergoing diagnostic or interventional coronary angiography and ascertained whether there was an association of IFG with angiographically confirmed CAD, with the extent of atherosclerosis in CAD patients, and/or with the occurrence of myocardial infarction (MI) among CAD cases.

\section{SUBJECTS AND METHODS}

\section{Subjects}

The patients scheduled to undergo clinically indicated diagnostic or therapeutic coronary catheterization at the First Affiliated Hospital of Shantou University Medical College during the period from 1 January 2009 to 31 December 2013 were recruited in this study. Patients who were currently pregnant or under taking fasting plasma glucose-lowering or steroid hormones medication or with history of chronic kidney disease and diabetes were excluded. A total of 1,451 nondiabetic individuals were enrolled in this study. All subjects were Chinese and older than 18 years. The study was approved by the research ethics committee of the First Affiliated Hospital of Shantou University Medical College.

Demographic and clinical data including age, sex, total cholesterol (TC) level, low-density-lipoprotein-cholesterol (LDLc) level, high-density-lipoprotein-cholesterol (HDLc) level, triglycerides level, blood pressure, coronary angiographic findings, and prevalent or incident MI were collected from hospital records. Blood samples for TC, LDLc, HDLc, triglycerides and fasting blood glucose (FBG) measurements were collected after overnight fasting. TC, LDLc, HDLc, triglycerides and FBG measurements were conducted by the clinical chemistry department of the First Affiliated Hospital of Shantou University Medical College. Coronary angiography was carried out by experienced interventional cardiologists. CAD was defined as $\geq 50 \%$ diameter stenosis in any of the major epicardial coronary artery, as measured by quantitative coronary angiography (Douglas et al., 2011; Dehmer et al., 2012; Fihn et al., 20142014). Disease severity was determined by number of diseased vessels (Golbahar et al., 2005). In this study, myocardial infarction included acute myocardial infarction and history of myocardial infarction. A diagnosis of acute myocardial infarction was established when there is acute myocardial injury with clinical evidence of acute myocardial ischemia and with detection of a rise and/or fall of cTn values with at least one value above the 99th percentile URL and at least one of the following: symptoms of myocardial ischemia; new ischemic ECG changes; development of pathological Q waves; imaging evidence of new loss of viable myocardium or new regional wall motion abnormality in a pattern consistent with an ischemic etiology; identification of a coronary thrombus by angiography. (Thygesen et al., 2018) IFG was defined by a fasting glucose level of $5.61-6.99 \mathrm{mmol} / \mathrm{L}$ and non-IFG by $\leq$ $5.60 \mathrm{mmol} / \mathrm{L}$.

\section{Statistical Analysis}

Data were analyzed using SPSS software, version 21.0 (SPSS Inc., Chicago, IL). The T-test and analysis of variance (ANOVA) were used to determine any differences in age, systolic blood pressure, diastolic blood pressure, TC level, LDLc level, HDLc level, and triglycerides level, between study groups. The $\chi^{2}$ test was performed to compare male/female ratio between groups and to compare the presence/absence of IFG between groups. Logistic regression analyses were performed to ascertain a relationship between IFG and the number of coronary artery with $\geq 50 \%$ stenosis among CAD cases with or without adjustment for age, TC and LDLc, and to test a relationship between IFG and MI among CAD cases with or without adjustment for sex, systolic blood pressure, diastolic blood pressure and triglyceride. All $\mathrm{p}$-values are two sided.

\section{RESULTS}

Demographic and clinical characteristics of the study subjects are shown in Table 1

The prevalence of IFG (fasting blood glucose level 5.61-6.99 mmol/L) was higher in patients with angiographically confirmed CAD ( $\geq 50 \%$ stenosis in at least 1 coronary artery) than in individuals without angiographic evidence of CAD (33.4 versus 28.2\%, $p=0.034$ without adjustment for co-variants, $p=0.044$ with adjustment for sex, age and HDLc level).

Compared with CAD cases without IFG, CAD cases with IFG had a higher odds ratio (OR) of having triple-vessel disease ( $\geq 50 \%$ stenosis in 3 coronary arteries) as opposed to having single- or double-vessel disease ( $\geq 50 \%$ stenosis in just 1 or 2 coronary arteries) $[\mathrm{OR}=1.53,95 \%$ confidence interval $(\mathrm{CI})=$ 1.13-2.07], which remained significant after adjusting age, total cholesterol level, and LDLc level $(\mathrm{OR}=1.52,95 \% \mathrm{CI}=1.10-2.10)$ (Table 2).

Furthermore, the occurrence of MI was higher in CAD cases with IFG than in CAD cases without IFG (OR $=1.73,95 \% \mathrm{CI}=$ 1.27-2.36), which remained significant after adjustment for sex, age, systolic blood pressure, diastolic blood pressure and triglyceride $)(\mathrm{OR}=1.79,95 \% \mathrm{CI}=1.28-2.50)$ (Table 3$)$.

\section{DISCUSSION}

In our study, we found that the prevalence of IFG was higher in patients with angiographically confirmed CAD than in subjects without angiographic evidence of CAD. It was in agreement with previous study reporting and several meta-analysis 
TABLE 1 | Demographic and clinical characteristics of study subjects.

\begin{tabular}{|c|c|c|c|c|c|c|c|c|}
\hline & \multicolumn{5}{|c|}{ Number of coronary artery with $\geq \mathbf{5 0} \%$ stenosis } & \multicolumn{3}{|c|}{ Occurrence of $M I$ in CAD cases ${ }^{a}$} \\
\hline & $0(n=586)$ & $\begin{array}{c}1 \text { or } 2 \\
(n=601)\end{array}$ & $3(n=264)$ & $p$-value ${ }^{b}$ & $p$-value ${ }^{c}$ & No $(n=296)$ & Yes $(n=569)$ & $p$-value \\
\hline Sex (male/female) & $\begin{array}{c}321 / 265 \\
(54.8 \% / 45.2 \%)\end{array}$ & $\begin{array}{c}484 / 117 \\
(80.5 \% / 19.5 \%)\end{array}$ & $\begin{array}{c}216 / 48 \\
(81.8 \% / 18.2)\end{array}$ & $<0.001$ & 0.658 & $\begin{array}{c}218 / 78 \\
(73.6 \% / 26.4 \%)\end{array}$ & $\begin{array}{c}482 / 87 \\
(84.7 \% / 15.3 \%)\end{array}$ & $<0.001$ \\
\hline Age (years) & $58.25 \pm 10.86$ & $62.10 \pm 11.18$ & $65.59 \pm 10.67$ & $<0.001$ & $<0.001$ & $63.64 \pm 10.54$ & $62.92 \pm 11.44$ & 0.358 \\
\hline $\begin{array}{l}\text { Systolic blood pressure } \\
(\mathrm{mmHg})\end{array}$ & $135.54 \pm 21.38$ & $134.15 \pm 24.61$ & $133.44 \pm 23.00$ & 0.193 & 0.679 & $141.02 \pm 22.34$ & $130.24 \pm 24.20$ & $<0.001$ \\
\hline $\begin{array}{l}\text { Diastolic blood pressure } \\
(\mathrm{mmHg})\end{array}$ & $83.17 \pm 13.56$ & $82.07 \pm 14.21$ & $81.31 \pm 13.30$ & 0.070 & 0.447 & $84.07 \pm 13.29$ & $80.68 \pm 14.13$ & 0.001 \\
\hline Total cholesterol (mmol/L) & $4.91 \pm 1.18$ & $4.86 \pm 1.24$ & $5.19 \pm 1.45$ & 0.445 & 0.001 & $5.01 \pm 1.45$ & $4.94 \pm 1.24$ & 0.474 \\
\hline $\begin{array}{l}\text { Low density lipoprotein } \\
(\mathrm{mmol} / \mathrm{L})\end{array}$ & $3.07 \pm 0.96$ & $3.06 \pm 1.04$ & $3.33 \pm 1.15$ & 0.176 & 0.002 & $3.14 \pm 1.17$ & $3.15 \pm 1.04$ & 0.830 \\
\hline $\begin{array}{l}\text { High density lipoprotein } \\
(\mathrm{mmol} / \mathrm{L})\end{array}$ & $1.17 \pm 0.33$ & $1.13 \pm 0.30$ & $1.12 \pm 0.27$ & 0.006 & 0.727 & $1.15 \pm 0.30$ & $1.12 \pm 0.29$ & 0.137 \\
\hline Triglyceride (mmol/L) & $1.56 \pm 1.03$ & $1.60 \pm 1.05$ & $1.51 \pm 0.88$ & 0.227 & 0.510 & $1.72 \pm 1.25$ & $1.49 \pm 0.83$ & 0.019 \\
\hline $\begin{array}{l}\text { Impaired fasting glucose } \\
\text { (Yes/No) }\end{array}$ & $\begin{array}{c}165 / 421 \\
(28.2 \% / 71.8 \%)\end{array}$ & $\begin{array}{c}183 / 418 \\
(30.4 \% / 69.6 \%)\end{array}$ & $\begin{array}{c}106 / 158 \\
(40.2 \% / 59.8 \%)\end{array}$ & 0.034 & 0.005 & $\begin{array}{c}76 / 220 \\
(25.7 \% / 74.3 \%)\end{array}$ & $\begin{array}{c}213 / 356 \\
(37.4 \% / 62.6 \%)\end{array}$ & 0.001 \\
\hline
\end{tabular}

Continuous variables are presented as mean \pm standard deviation, and categorical variables are expressed as frequency and percentage.

${ }^{a}$ Referred to cases with $\geq 50 \%$ stenosis in at least 1 coronary artery.

${ }^{d}$ Fasting blood glucose level $=5.61-6.99 \mathrm{mmol} / \mathrm{L}$.

${ }^{b}$ Comparing subject without $\geq 50 \%$ stenosis to subjects with $\geq 50 \%$ stenosis in at least 1 coronary artery.

${ }^{c}$ Comparing subject with $\geq 50 \%$ stenosis in 1 or 2 coronary arteries to subjects with $\geq 50 \%$ stenosis in 3 coronary arteries.

TABLE 2 | Impaired fasting blood glucose and extent of coronary artery disease.

\begin{tabular}{|c|c|c|c|c|}
\hline \multirow[t]{2}{*}{ Impaired fasting glucose } & \multirow{2}{*}{$\begin{array}{c}\begin{array}{c}\text { Numbers of coronary } \\
\text { artery with } \geq 50 \% \\
\text { stenosis }\end{array} \\
1 \text { or } 2\end{array}$} & \multirow{2}{*}{$\frac{\text { Odds ratio }(95 \% \mathrm{Cl})}{3}$} & \multirow{2}{*}{\multicolumn{2}{|c|}{ Adjusted odds ratio $(95 \% \mathrm{Cl})^{a}$}} \\
\hline & & & & \\
\hline $\mathrm{No}^{\mathrm{b}}$ & 418 (69.6\%) & $158(59.8 \%)$ & 1.00 (reference) & 1.00 (reference) \\
\hline Yes $^{c}$ & 183 (30.4\%) & 106 (40.2\%) & 1.53 (1.13-2.07) & 1.52 (1.10-2.10) \\
\hline
\end{tabular}

${ }^{a}$ Logistic regression analysis with adjustment for age, total cholesterol level and Low density lipoprotein.

${ }^{b}$ Fasting blood glucose level $\leq 5.60 \mathrm{mmol} / \mathrm{L}$.

${ }^{c}$ Fasting blood glucose level $5.61-6.99 \mathrm{mmol} / \mathrm{L}$.

TABLE 3 | Impaired fasting glucose and myocardial infarction In patients with $\geq 50 \%$ stenosis in at least one coronary artery.

\begin{tabular}{|c|c|c|c|c|}
\hline \multirow[t]{2}{*}{ Impaired fasting glucose } & \multicolumn{2}{|c|}{ Myocardial infarction } & \multirow[t]{2}{*}{ Odds ratio $(95 \% \mathrm{Cl})$} & \multirow{2}{*}{$\begin{array}{l}\text { Adjusted odds ratic } \\
(95 \% \mathrm{Cl})^{\mathrm{a}}\end{array}$} \\
\hline & No & Yes & & \\
\hline $\mathrm{No}^{\mathrm{b}}$ & $220(38.2 \%)$ & $356(61.8 \%)$ & 1.00 (reference) & 1.00 (Reference) \\
\hline Yes $^{\mathrm{c}}$ & 76 (26.3\%) & $213(73.7 \%)$ & $1.73(1.27-2.36)$ & $1.79(1.28-2.50)$ \\
\hline
\end{tabular}

aLogistic regression analysis with adjustment for sex, systolic blood pressure, diastolic blood pressure and triglyceride level.

${ }^{b}$ Fasting blood glucose level $\leq 5.60 \mathrm{mmol} / \mathrm{L}$.

${ }^{c}$ Fasting blood glucose level $5.61-6.99 \mathrm{mmol}$

(Konstantinou et al., 2010; Xu et al., 2015; Huang et al., 2016; Cai et al., 2020).

Previous studies had shown that prediabetes were associated with an increased risk of heart failure (HF), and a worse prognosis with HF. (Cai et al., 2021; Mai et al., 2021) Also, In our study, we found that compared with $\mathrm{CAD}$ cases without IFG, CAD cases with IFG were suffered from triple-vessel stenosis more than single- or double-vessel stenosis. It may be suggested that IFG promoted involvement of coronary arteries with a more aggressive and diffused atherosclerotic process and had a worse prognosis.

It is still unclear how IFG promoted the progress of CAD. Hyperglycemia, vascular insulin resistance and other inflammatory cytokines contributed to endothelial vasodilator dysfunction and increased formation of Extracellular Matrix in IFG patients (Wasserman et al., 2018). Advanced glycation products (AGEs) stimulated monocytes to secrete inflammatory cytokines and result in the formation of foam 
cells (Vlassara et al., 1988; Jinnouchi et al., 1998; Wasserman et al., 2018). All of these factors may contribute to progression of the triple-vessel disease in IFG patients.

Several limitations should be considered. Firstly, the crosssectional nature of the study prevents us from confirming the causal effects angiographically confirmed CAD cases in the pathogenesis of IFG. Secondly, triple-vessel disease often indicates a poor prognosis for patients. However, in this study, we did not observe the prognosis of the patients which limited its persuasion. Third, not only IFG but also impaired glucose tolerance (IGT), plays important role in CAD progression (Huang et al., 2016). However, in the study, we did not include IGT which need to be further investigated.

\section{DATA AVAILABILITY STATEMENT}

The raw data supporting the conclusions of this article will be made available by the authors, without undue reservation.

\section{ETHICS STATEMENT}

The studies involving human participants were reviewed and approved by research ethics committee of the First Affiliated Hospital of Shantou University Medical College. Written informed consent for participation was not required for this study in accordance with the national legislation and the

\section{REFERENCES}

Cai, X., Liu, X., Sun, L., He, Y., Zheng, S., Zhang, Y., et al. (2021). Prediabetes and the Risk of Heart Failure: A Meta-analysis. Diabetes Obes. Metab. 23 (8), 1746-1753. doi:10.1111/dom.14388

Cai, X., Zhang, Y., Li, M., Wu, J. H., Mai, L., Li, J., et al. (2020). Association between Prediabetes and Risk of All Cause Mortality and Cardiovascular Disease: Updated Meta-Analysis. Bmj 370, m2297. doi:10.1136/bmj.m2297

Dehmer, G. J., Weaver, D., Roe, M. T., Milford-Beland, S., Fitzgerald, S., Hermann, A., et al. (2012). A Contemporary View of Diagnostic Cardiac Catheterization and Percutaneous Coronary Intervention in the United States. J. Am. Coll. Cardiol. 60 (20), 2017-2031. doi:10.1016/j.jacc.2012.08.966

Douglas, P. S., Patel, M. R., Bailey, S. R., Dai, D., Kaltenbach, L., Brindis, R. G., et al. (2011). Hospital Variability in the Rate of Finding Obstructive Coronary Artery Disease at Elective, Diagnostic Coronary Angiography. J. Am. Coll. Cardiol. 58 (8), 801-809. doi:10.1016/j.jacc.2011.05.019

Fihn, S. D., Blankenship, J. C., Alexander, K. P., Bittl, J. A., Byrne, J. G., Fletcher, B. J., et al. (20142014). 2014 ACC/AHA/AATS/PCNA/SCAI/STS Focused Update of the Guideline for the Diagnosis and Management of Patients with Stable Ischemic Heart Disease. J. Am. Coll. Cardiol. 64 (18), 1929-1949. doi:10.1016/j. jacc.2014.07.017

Golbahar, J., Aminzadeh, M. A., Sharifkazemi, M. B., and Rezaian, G. R. (2005). Association of Red Blood Cell 5-methyltetrahydrofolate and Severity of Coronary Artery Disease: a Cross-Sectional Study from Shiraz, Southern Iran. Heart Vessels 20 (5), 203-206. doi:10.1007/s00380-004-0823-3

Hanna-Moussa, A., Gardner, M. J., Kurukulasuriya, L. R., and Sowers, J. R. (2009). Dysglycemia/prediabetes and Cardiovascular Risk Factors. Rev. Cardiovasc. Med. 10 (4), 202-208. doi:10.3909/ricm0474

Henry, P., Thomas, F., Benetos, A., and Guize, L. (2002). Impaired Fasting Glucose, Blood Pressure and Cardiovascular Disease Mortality. Hypertension 40 (4), 458-463. doi:10.1161/01.hyp.0000032853.95690.26 institutional requirements. Written informed consent was obtained from the individual(s) for the publication of any potentially identifiable images or data included in this article.

\section{AUTHOR CONTRIBUTIONS}

XT and SY performed the survey and wrote the draft of manuscript. CC, YC, and JX designed the study and collected data. YZ, ZY, PY, NL, and KY helped interpret the analysis results, XC, SC; MO'G and MO'M assisted in modifying the manuscript. All authors read and approved the final manuscript.

\section{FUNDING}

This work was supported by the National Natural Science Foundation of China (No. 30771836), the Natural Science Foundation of Guangdong Province China (2015A030310040), the Medical Scientific Research Foundation of Guangdong Province of China (A2008440), 2014 Shantou Finance and Education Projects (No.242), 2015 Shantou Science and Technology Projects (No.123), 'Dengfeng' Project for the construction of high-level hospitals in Guangdong Provincethe First Affiliated Hospital of Shantou University Medical College Supporting Funding(No. 202003-2), and Special project for high-level hospital construction(No. 210716096900364)

Huang, Y., Cai, X., Mai, W., Li, M., and Hu, Y. (2016). Association between Prediabetes and Risk of Cardiovascular Disease and All Cause Mortality: Systematic Review and Meta-Analysis. Bmj 355, i5953. doi:10.1136/bmj. i5953

Jinnouchi, Y., Sano, H., Nagai, R., Hakamata, H., Kodama, T., Suzuki, H., et al. (1998). Glycolaldehyde-modified Low Density Lipoprotein Leads Macrophages to Foam Cells via the Macrophage Scavenger Receptor. J. Biochem. 123 (6), 1208-1217. doi:10.1093/oxfordjournals.jbchem.a022062

Kannel, W. B., and McGee, D. L. (1979). Diabetes and Cardiovascular Disease. The Framingham Study. Jama 241 (19), 2035-2038. doi:10.1001/jama.241.19. 2035

Konstantinou, D. M., Chatzizisis, Y. S., Louridas, G. E., Parcharidis, G. E., and Giannoglou, G. D. (2010). Non-diabetic Hyperglycaemia Correlates with Angiographic Coronary Artery Disease Prevalence and Severity. Diabetes Metab. 36 (5), 402-408. doi:10.1016/j.diabet.2010.04.005

Mai, L., Wen, W., Qiu, M., Liu, X., Sun, L., Zheng, H., et al. (2021). Association between Prediabetes and Adverse Outcomes in Heart Failure. Diabetes Obes. Metab. 23 (11), 2476-2483. doi:10.1111/dom.14490

Martins, J., Olorunju, S. A. S., Murray, L. M., and Pillay, T. S. (2015). Comparison of Equations for the Calculation of LDL-Cholesterol in Hospitalized Patients. Clinica Chim. Acta 444, 137-142. doi:10.1016/j.cca.2015.01.037

Sorkin, J. D., Muller, D. C., Fleg, J. L., and Andres, R. (2005). The Relation of Fasting and 2-h Postchallenge Plasma Glucose Concentrations to Mortality. Diabetes Care 28 (11), 2626-2632. doi:10.2337/diacare.28.11.2626

Thygesen, K., Alpert, J. S., Jaffe, A. S., Chaitman, B. R., Bax, J. J., Morrow, D. A., et al. (2018). Fourth Universal Definition of Myocardial Infarction (2018). J. Am. Coll. Cardiol. 72 (18), 2231-2264. doi:10.1016/j.jacc.2018. 08.1038

Vlassara, H., Brownlee, M., Manogue, K. R., Dinarello, C. A., and Pasagian, A. (1988). Cachectin/TNF and IL-1 Induced by Glucose-Modified Proteins: Role in normal Tissue Remodeling. Science 240 (4858), 1546-1548. doi:10.1126/ science. 3259727 
Wasserman, D. H., Wang, T. J., and Brown, N. J. (2018). The Vasculature in Prediabetes. Circ. Res. 122 (8), 1135-1150. doi:10.1161/circresaha.118.311912

Xu, T., Liu, W., Cai, X., Ding, J., Tang, H., Huang, Y., et al. (2015). Risk of Coronary Heart Disease in Different Criterion of Impaired Fasting Glucose. Medicine (Baltimore) 94 (40), e1740. doi:10.1097/md.0000000000001740

Yan, Q., Gu, W. Q., Hong, J., Zhang, Y. F., Su, Y. X., Gui, M. H., et al. (2009). Coronary Angiographic Studies of Impaired Glucose Regulation and Coronary Artery Disease in Chinese Nondiabetic Subjects. Endocr 36 (3), 457-463. doi:10. 1007/s12020-009-9261-4

Conflict of Interest: The authors declare that the research was conducted in the absence of any commercial or financial relationships that could be construed as a potential conflict of interest.
Publisher's Note: All claims expressed in this article are solely those of the authors and do not necessarily represent those of their affiliated organizations, or those of the publisher, the editors and the reviewers. Any product that may be evaluated in this article, orclaim that may be made by its manufacturer, is not guaranteed or endorsed by the publisher.

Copyright $\odot 2022$ Chen, Chen, Xiao, Zhang, Yang, Yang, Lu, Yi, Chen, Chen, O'Gara MSc, O'Meara, Ye and Tan. This is an open-access article distributed under the terms of the Creative Commons Attribution License (CC BY). The use, distribution or reproduction in other forums is permitted, provided the original author(s) and the copyright owner(s) are credited and that the original publication in this journal is cited, in accordance with accepted academic practice. No use, distribution or reproduction is permitted which does not comply with these terms. 